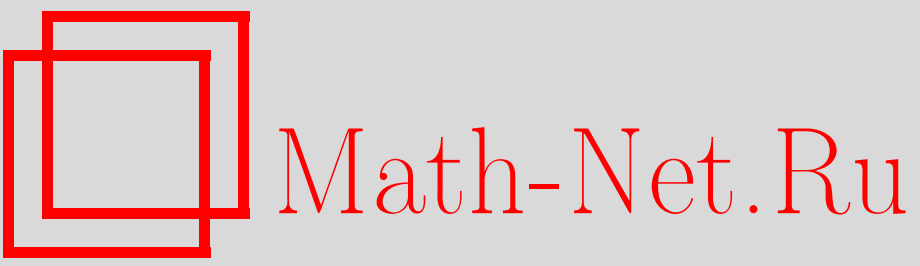

Б. С. Павлов, В. И. Рыжий, Точечные и антиточечные квантовые инфракрасные детекторы: итерационные методы решения уравнения Лапласа в сложных областях, ТМФ, 2004, том 141, номер 2, 163-177

DOI: https://doi.org/10.4213/tmf123

Использование Общероссийского математического портала Math-Net.Ru подразумевает, что вы прочитали и согласны с пользовательским соглашением

http://www.mathnet.ru/rus/agreement

Параметры загрузки:

IP : 54.197 .217 .227

26 апреля 2023 г., 17:18:23 


\section{ТОЧЕЧНЫЕ И АНТИТОЧЕЧНЫЕ КВАНТОВЫЕ ИНФРАКРАСНЫЕ ДЕТЕКТОРЫ: ИТЕРАЦИОННЫЕ МЕТОДЫ РЕШЕНИЯ УРАВНЕНИЯ ЛАПЛАСА В СЛОЖНЫХ ОБЛАСТЯХ}

Предложены итерационные методы для решения задачи Дирихле в сложных областях. Эти задачи возникают в связи с проблемой оптимизации точечных и антиточечных квантовых инфракрасных детекторов. Дана оценка приближенного решения относительно точного.

Ключевые слова: гармонические функции, отображение Пуассона, принцип максимума.

\section{1. ВВЕДЕНИЕ}

Квантово-точечный инфракрасный фотодетектор (КТИФ) может быть построен (см., например, [1], [2]) как набор квантовых точек, вкрапленных в материал с относительно широкой полосой энергетической лакуны. Эта структура помещается между контактными слоями: эмиттером $\Gamma_{+}: z=L$ и коллектором $\Gamma_{-}: z=0$. Проводимость всей структуры зависит от распределения потенциала $\varphi(x, y, z)$ между контактными слоями, которое определяется напряжением, приложенным к ним, и зарядами квантовых точек или зарядовой плотностью на пластинах, содержаших антиточки. В частности, плотность тока фотоэмиссии на эмиттере вычисляется как

$$
j_{\text {photo }}(x, z)=j_{\max } \exp \left(\frac{e \varphi(x, y, L)}{k_{\mathrm{B}} T}\right)
$$

где $j_{\max }$ - максимальная плотность тока, $e \varphi(x, y, L)$ - произведение заряда электрона на потенциал эмиттера, $k_{\mathrm{B}}$ - постоянная Больцмана, $T$ - абсолютная температура. С другой стороны, распределение заряда определяется процессом фотоэмиссии из точек, и проводимость антиточечного устройства зависит от распределения потенциала в

*Department of Mathematics, University of Auckland, Auckland, New Zealand. E-mail: pavlov@math.auckland.ac.nz

${ }^{\dagger}$ Solid State Computer Laboratory, University of Aizu, Aizu-Wakamatsu-City, Japan. E-mail: v-ryzhii@u-aizu.ac.jp 
объеме материала полупроводника и определяется интенсивностью фотоэмиссии с металлических пластин. В обоих случаях потенциал может быть посчитан как решение соответствующей задачи Дирихле для уравнения Лапласа в области со сложной геометрией, как предложено в работе [3]. Если геометрические параметры области уже выбраны, численное решение возможно. Но из-за присутствия многочисленных геометрических параметров оптимизация соответствуюшего построения по-прежнему трудна. Мы предлагаем итерационные методы для обеих задач Дирихле с точечным и антиточечным облачением и представляем надлежашую оценку отклонения приближенного решения от соответствуюшего точного. Построенное приближенное решение содержит все геометрические параметры в точной форме и, таким образом, может быть подставлено для получения точного решения в целях оптимизации построения КТИФ или антиточечного инфракрасного фотодетектора.

\section{2. ИТЕРАЦИОННОЕ РЕШЕНИЕ ГРАНИЧНОЙ ЗАДАЧИ ДЛЯ УРАВНЕНИЯ ЛАПЛАСА В ПРИСУТСТВИИ КВАНТОВОЙ ТОЧКИ}

Идея построения итерационного решения, основанная на принципе максимума для гармонических функций, вдохновлена книгой [4] и статьями [5], [6]. В случае КТИФ мы можем начать с построения функции Грина для слоя $\Omega$ между двумя контактными плоскостями $\Gamma_{ \pm}$и соответствуюшего отображения Пуассона $P$. Отображение Пуассона позволяет вычислить потенциал $u$ внутри слоя для любых, в частности периодических, граничных данных гармонической функции (потенциала) на контактных плоскостях $\Gamma_{ \pm}$. В действительности потенциал определяется также набором простых стандартных проводящих тел $\omega_{s}$, погруженных в слой $\Omega$ между плоскостями, как $\left.u\right|_{\partial \omega_{s}}=u_{\gamma_{s}}$. Эти тела играют роль квантовых точек и могут быть выбраны как эллипсоиды (сфероиды) или даже диски. Решение задачи Дирихле в дополнении к одиночному сфероидальному проводящему телу может быть получено путем разделения переменных в форме сходящегося ряда функций Ламе (см., например, [7]), но уже для двух тел разделение переменных становится невозможным. Мы утверждаем, что наш итерационный метод позволяет получить решение задачи Дирихле и в этом случае, и в значительно более трудном случае нескольких тел внутри слоя. Если тела распределены периодически, то задача во всем слое в присутствии тел сводится к задаче на периоде.

Сушественным наблюдением является оценка согласно принципу максимума для решения $u$ задачи Дирихле в слое для единичного тела $\omega$ с нулевыми граничными условиями на контактных плоскостях; эту оценку следует производить в терминах решения $U$ задачи Дирихле в дополнении $\mathbb{R}^{3} \backslash \omega$ с теми же данными на границе $\gamma=\partial \omega$ тела $\omega$.

Опишем алгоритм построения решения. Рассмотрим отображение Пуассона $P_{\omega}$ в дополнении к $\omega$ и решение

$$
u_{1}(x)=\left.P_{\omega} u\right|_{\gamma}=P_{\omega} u_{\gamma}
$$

задачи Дирихле в $\mathbb{R}^{3} \backslash \omega$ с граничными данными $\left.u\right|_{\gamma}=u_{\gamma}$ на $\gamma$. Если $U(x)=P_{\omega} \chi$ - гармоническая функция на дополнении к $\omega$ со стандартными граничными данными 
$\left.U_{s}(x)\right|_{\gamma}=\chi=1$, то в силу принципа максимума

$$
|u(x)| \leqslant U(x) \max _{\gamma}\left|u_{\gamma}\right|
$$

Обозначая через $\left.u\right|_{\Gamma}$ сужение решения $u$ на сумму $\Gamma=\Gamma_{+} \cup \Gamma_{-}$контактных плоскостей $\Gamma_{ \pm}$, мы можем рассмотреть “отраженное” решение $P\left[\left.u\right|_{\Gamma}\right]=u_{\gamma}^{(1)}$ внутри слоя $\Omega$. Гармоническая функция

$$
u_{\gamma}^{1}=u-u_{\gamma}^{(1)}
$$

равна нулю на контактных плоскостях, и сужение ее на границу $\Gamma \cup \gamma$ может быть оценено по принципу максимума:

$$
\left.\left|u_{\gamma}^{(1)}\right|\right|_{\gamma} \leqslant\left. U\right|_{\Gamma} \max _{\gamma}\left|u_{\gamma}\right|
$$

Тогда сужение “дважды отраженного” решения

$$
P_{\omega}\left[\left.u_{\gamma}^{(1)}\right|_{\gamma}\right]=u_{\gamma}^{(2)}
$$

на контактные плоскости удовлетворяет оценке

$$
\left.u_{\gamma}^{(2)}\right|_{\Gamma} \leqslant\left.[U]^{2}\right|_{\Gamma} \max _{\gamma}\left|u_{\gamma}\right|
$$

Гармоническая функция

$$
u_{3}=u(x)-u^{(1)}(x)+u^{(2)}
$$

совпадает с граничной функцией $\left.u\right|_{\gamma}$ на $\partial \gamma$ и оценивается на контактных плоскостях как

$$
\max \left|u_{3}\right| \Gamma\left|\leqslant \max _{\Gamma}[U]\right|_{\Gamma} \max _{\Gamma}\left|u_{1}\right|
$$

Таким образом, преобразующий граничные данные оператор

$$
T:\left.\left.u_{1}\right|_{\Gamma} \longrightarrow u_{3}\right|_{\Gamma}
$$

является сжимающим с нормой, оцениваемой как

$$
\|T\| \leqslant\left.\max _{\Gamma}[U]\right|_{\Gamma}=\kappa<1
$$

в пространстве $C_{\Gamma_{+}} \cup \Gamma_{-}$непрерывных функций на контактных плоскостях. Тогда итерации

$$
u_{1} \rightarrow T u_{1}=u_{2} \rightarrow T u_{2}=T^{2} u_{1}=u_{5} \rightarrow \cdots
$$

образуют геометрически сходящуюся последовательность с общим членом $u_{2 s+1}$, оцениваемым на $\Gamma_{+} \cup \Gamma_{-}$как

$$
\max _{\Gamma}\left|u_{s}\right| \leqslant \kappa^{s} \max _{\Gamma}\left|u_{1}\right|
$$


Следовательно, построенная последовательность итераший сходится к решению задачи Дирихле на $\gamma$ с граничными данными

$$
\left.u\right|_{\gamma}=u_{\gamma},\left.\quad u\right|_{\Gamma}=0
$$

так что соответствующее отображение Пуассона $\mathcal{P}_{\gamma}$ может быть получено как сумма геометрически сходящейся последовательности

$$
\mathcal{P}_{\gamma}=P_{\gamma} *-\left.P\left[P_{\gamma} *\right]\right|_{\Gamma}+\left.P_{\gamma}\left[\left.P\left[P_{\gamma} *\right]\right|_{\Gamma}\right]\right|_{\gamma}-\left.P\left[\left.P_{\gamma}\left[\left.P\left[P_{\gamma} *\right]\right|_{\Gamma}\right]\right|_{\gamma}\right]\right|_{\Gamma}-\cdots
$$

Теперь мы можем построить "дополнительное" отображение Пуассона $\mathcal{P}_{\Gamma}$ в "возмушенной” области $\Omega \backslash \omega$, которое может быть использовано для построения решения граничной задачи с ненулевыми данными только на $\Gamma$. Мы можем построить $\mathcal{P}_{\Gamma}$, основываясь на отображении Пуассона $P$ в невозмушенной области $\Omega$ и пользуясь уже вычисленным “суженным" отображением Пуассона $\mathcal{P}_{\gamma}$ :

$$
\mathcal{P}_{\Gamma} *=\left.P *\right|_{\Gamma}-\left.\mathcal{P}_{\gamma}\left[\left.P *\right|_{\Gamma}\right]\right|_{\gamma}
$$

Тогда сумма $\mathcal{P}_{\Gamma}+\mathcal{P}_{\gamma}=\mathcal{P}$ является отображением Пуассона с возмущенной области $\Omega \backslash \omega$.

Построение итерационного решения в случае нескольких тел $\omega_{s}$ в области $\Omega$ можно выполнить по индукции, используя полученное на первом шаге отображение Пуассона $\mathcal{P}=\mathcal{P}_{1}$ в $\Omega \backslash \omega$ вместо отображения Пуассона $P$ в "пустом" слое $\Omega$. Затем, добавляя второе тело $\omega_{2}$, как ранее, мы получаем итерации отображения Пуассона $\mathcal{P}_{2}$ для области с двумя телами $\omega=\omega_{1}, \omega_{2}$ и т.д. Если второе тело находится далеко от первого, то возмушение отображения Пуассона $\mathcal{P}_{2}-\mathcal{P}_{1}$, вызванное добавлением второго тела, может быть оценено по квадрату обратного расстояния $\left|1 / d_{12}\right|$ между телами в любой подобласти $\Omega_{1}, \operatorname{diam} \Omega_{1} \leqslant d_{12} / 2$. В конечном итоге это позволяет распространить итерационную процедуру на периодическое распределение квантовых точек.

Описанная процедура построения итерационного решения может быть интерпретирована как решение интегрального уравнения. Предположим, что отображения Пуассона $\mathcal{P}_{s}, \quad s=1,2$, в дополнениях $\mathbb{R}^{3} \backslash \omega_{s}:=\Omega_{s}$ известны. Построим решение задачи Дирихле в области $\Omega=\mathbb{R}^{3} \backslash\left(\omega_{1} \cup \omega_{2}\right)$ с граничными условиями на обшей границе $\partial \Omega=\partial \omega_{1} \cup \partial \omega_{2}:=\Gamma_{1} \cup \Gamma_{2}$ :

$$
\left.u\right|_{\Gamma_{1}}=u_{1},\left.\quad u\right|_{\Gamma_{2}}=u_{2} .
$$

Не ограничивая обшности, можно положить, что $u_{1} \in C_{\Gamma_{1}}$ не равно тождественно нулю, но $u_{2}=0$. Предположим, что решения $\xi_{s}, s=1,2$, задачи Дирихле в области $\Omega_{s}, s=$ 1,2, с граничными условиями

$$
\left.\xi_{s}\right|_{\Gamma_{s}}=1
$$

построены и $\left.\sup \xi_{s}\right|_{\Gamma_{t}}<1$ по крайней мере для одной комбинации $s=1, t=2$ или $s=2$, $t=1$. 
Теорема 1. Решение задачи Дирихле в области $\Omega$ для уравнения Лапласа с граничными условиями (2) может быть выражено в форме

$$
\mathcal{P}_{1} u-\left.\mathcal{P}_{2}\left[\mathcal{P}_{1} u\right]\right|_{\Gamma_{2}},
$$

где $и$-решение интегрального уравнения в $C_{\Gamma_{1}}$ :

$$
u-\left.\left[\left.\mathcal{P}_{2}\left[\mathcal{P}_{1} u\right]\right|_{\Gamma_{2}}\right]\right|_{\Gamma_{1}}=u_{1}
$$

Это интегральное уравнение имеет единственное решение в $C_{\Gamma_{1}}$, поскольку интегральный оператор

$$
K:\left.u \rightarrow\left[\left.\mathcal{P}_{2}\left[\mathcal{P}_{1} u\right]\right|_{\Gamma_{2}}\right]\right|_{\Gamma_{1}}
$$

является сжатием при выполнении условия $\left.\sup \xi_{s}\right|_{\Gamma_{t}}<1$ при $s=1, \quad t=2$ или $s=2, t=1$ :

$$
\|K\|_{C_{\Gamma_{1}}} \leqslant\left.\left.\sup \xi_{1}\right|_{\Gamma_{2}} \sup \xi_{2}\right|_{\Gamma_{1}}
$$

ДокАЗАТЕЛЬСТво основано на принципе максимума:

$$
\left|\mathcal{P}_{1} u_{1}\right|_{\Gamma_{2}} \leqslant \xi\left\|u_{1}\right\|_{C_{\Gamma_{1}}} .
$$

\section{3. ИТЕРАЦИОННОЕ РЕШЕНИЕ ГРАНИЧНОЙ ЗАДАЧИ ДЛЯ УРАВНЕНИЯ ЛАПЛАСА В ПРИСУТСТВИИ АНТИТОЧЕЧНОГО РАСПРЕДЕЛЕНИЯ}

Начнем с трехмерного уравнения Лапласа

$$
-\Delta u=0
$$

в бесконечном слое $\Omega$ : $0<z<H$ в вакууме, разделенном "металлическими" плоскостями $L_{s}: H_{s-1}<z<H_{s}, H_{0}=0, H_{n}=d$, с граничными условиями на каждой плоскости

$$
\left.u\right|_{L_{s}}=u_{s}=\mathrm{const}, \quad s=0,1, \ldots, n \text {. }
$$

Эта “невозмущенная" граничная задача имеет единственное решение, представляемое кусочно-линейной функцией от одной переменной $z$

$$
u(z)=u_{s-1}+\left(z-H_{s-1}\right) \frac{u_{s}-u_{s-1}}{H_{s}-H_{s-1}}, \quad H_{s-1} \leqslant z \leqslant H_{s}, \quad s=1,2, \ldots, n,
$$

т.е. решение есть линейная функция от $z$ в каждом слое $\Omega_{s}: H_{s-1}<z<H_{s}$ с толшиной $H_{s}-H_{s-1}=d_{s}$ и принимает равные граничные значения $u_{s}$ на общей границе $L_{s}$ двух соседних слоев $\Omega_{s-1}, \Omega_{s}$. Скачок нормальной производной решения пропорционален зарядовой плотности $\rho$ на $L_{s}$ :

$$
\frac{u_{s+1}-u_{s}}{H_{s+1}-H_{s}}-\frac{u_{s}-u_{s-1}}{H_{s}-H_{s-1}}=4 \pi \rho .
$$


В настоящей статье мы рассматриваем периодические модификации указанной выше "невозмушенной" задачи. Они достигаются видоизменением основной области $\Omega \rightarrow \Omega$ путем выделения периодических дисковых структур $\omega_{s, t}$ радиуса $\delta$ на плоскостях $L_{s}$. Мы предполагаем, что "основная подобласть" периодической области $\boldsymbol{\Omega}$ - "период" $\boldsymbol{\Omega}_{0}$ - это параллелепипед $0<x_{1}<D_{1}, 0<x_{2}<D_{2}, 0<z<H$, который содержит конечный набор дисков $\omega_{s, t}$ на различных пластинах $\mathbf{L}_{s}=\Omega_{0} \cap L_{s}, 0<s<n, \bigcup_{t} \omega_{s, t}=\omega_{s}$. Обозначим дополнение $\mathbf{L}_{s} \backslash \boldsymbol{\omega}_{s}$ базового множсества дисков $\boldsymbol{\omega}_{s}$ на $\mathbf{L}_{s}$ через $\gamma_{s}$. Тогда полный слой $\boldsymbol{\Omega}$ с периодически распределенными дисками на пластинах может быть получен как счетная сумма сдвинутых копий $\boldsymbol{\Omega}_{m}=\left\{\mathbf{x}_{m}=\left[x_{1}+D_{1} m_{1}, x_{2}+D_{2} m_{2}\right]\right.$, $\left.m=\left(m_{1}, m_{2}\right) \in \mathbb{Z}_{2}, \mathbf{x}=\left(x_{1}, x_{2}\right) \in \boldsymbol{\Omega}_{0}\right\}$ периода $\boldsymbol{\Omega}_{0}$,

$$
\boldsymbol{\Omega}=\bigcup_{m \in \mathbb{Z}_{2}} \boldsymbol{\Omega}_{m}
$$

Сумму по всем периодическим сдвигам базового множества дисков на всей бесконечной пластине $L_{s}$ обозначим $\omega_{s}$, а ее дополнение на $L_{s}$ обозначим $\gamma_{s}$.

Мы исследуем периодическую граничную задачу в области $\boldsymbol{\Omega}$, которая может рассматриваться как возмушение исходной "невозмушенной” задачи:

Найти периодическое решение граничной задачи для уравнения Лапласа $\Delta u=0$ во всей области $\Omega$ с граничными данными

$$
\left.u\right|_{L_{0}}=0,\left.\quad u\right|_{\gamma_{s}}=u_{s}, \quad s=1,2, \ldots,\left.\quad u\right|_{L_{n}}=u_{n}
$$

и следующими условиями согласования на дисках: решения уравнения Лапласа в соседних слоях $\Omega_{s}, \Omega_{s+1}$ и их нормальные производные совпадают на дисках $\left\{\omega_{s, t}\right\}$.

Эта задача может быть предложена в качестве математической формулировки проблемы проводимости плазмы в присутствии периодического набора антиточек $\left\{\omega_{s, t}\right\}$.

Рассмотрим эквивалентную граничную задачу для уравнения Лапласа на возмущенном и невозмушенном (без “дыр”) периоде $\boldsymbol{\Omega}_{\mathbf{0}}$ с периодическими граничными условиями:

$$
\begin{aligned}
u\left(\xi_{1}+D_{1}, x_{2}\right) & =u\left(\xi_{1}, x_{2}\right), & u\left(x_{1}, \xi_{2}+D_{2}\right) & =u\left(x_{1}, \xi_{2}\right), \\
\nabla \partial u\left(\xi_{1}+D_{1}, x_{2}\right) & =\nabla u\left(\xi_{1}, x_{2}\right), & \nabla u\left(x_{1}, \xi_{2}+D_{2}\right) & =\nabla u\left(x_{1}, \xi_{2}\right),
\end{aligned}
$$

где точки $\left(\xi_{1}, x_{2}\right),\left(x_{1}, \xi_{2}\right)$ находятся на “вертикальной” границе основной области $\partial \boldsymbol{\Omega}_{0} \backslash$ $\left(L_{0} \cup L_{n}\right): \xi_{l}=0, D_{l}, 0<z<d, l=1,2$. В действительности конструкция решения граничной задачи на периоде может быть продолжена периодически с условиями (6) на всю область $\Omega$. Следовательно, мы можем ограничиться граничной задачей на периоде.

Начнем с построения ядра Пуассона в доле $\boldsymbol{\Omega}_{0, s}=\Omega_{s} \cap \boldsymbol{\Omega}_{0}$ каждого слоя $\Omega_{s}, s=$ $0,1, \ldots, n-1$, невозмушенной основной области $\boldsymbol{\Omega}_{0}$ (без дыр). Горизонтальные моды уравнения Лапласа в доле $\boldsymbol{\Omega}_{0, s}=\Omega_{s} \cap \boldsymbol{\Omega}_{0}$ есть нормированные экспоненты:

$$
\varphi_{l}=\frac{1}{\sqrt{D_{1} D_{2}}} e^{i\left[2 \pi x_{1} / D_{1}+2 \pi x_{2} / D_{2}\right]},
$$


следовательно, решение соответствуюшей граничной задачи в слое $d_{s-1}<z<d_{s}$ с (функциональными) граничными значениями $u_{s-1}(\mathbf{x}), u_{s}(\mathbf{x})$ на соответствующих долях $\mathbf{L}_{s-1}, \mathbf{L}_{s}$ плоскостей $L_{s-1}, L_{s}$ внутри периода может быть представлено как

$$
u_{s}(\mathbf{x}, z)=\sum_{l} \varphi_{l}(\mathbf{x})\left[u_{s-1, l} \frac{\operatorname{sh}|l|\left(H_{s}-z\right)}{\operatorname{sh}|l| d_{s}}+u_{s, l} \frac{\operatorname{sh}|l|\left(z-H_{s-1}\right)}{\operatorname{sh}|l| d_{s}}\right]
$$

с коэффициентами Фурье $u_{s, l}=\left\langle\varphi_{l}, u_{s}\right\rangle$ граничных данных $u_{s}$ касательно горизонтальных мод $\varphi_{l}$ в $L_{2}$ на соответствуюших горизонтальных секторах $\mathbf{L}_{s}$ основной области. Отображение граничных данных $u_{s-1}, u_{s}$ в периодические гармонические функции $u_{s}(\mathbf{x})$ в слое $\Omega_{s}$ называется отображением Пуассона и обозначается через $\mathbf{P}_{s}$. Его сужения на данные, поддерживаемые на нижней крышке $\mathbf{L}_{s-1}$ или верхней крышке $\mathbf{L}_{s}$ слоя $\boldsymbol{\Omega}_{s}$, будут обозначаться $\mathbf{P}_{s}^{ \pm}$, соответственно, так что, например, $\mathbf{P}_{s}^{+} u_{s-1} \mid \mathbf{L}_{s}=0$.

В следующем разделе мы используем вместе с отображениями Пуассона для периодических решений условные отображения Пуассона: $\mathcal{P}_{s}^{+}$в полупространстве $\Omega_{s}^{+}:(z-$ $\left.H_{s-1}>0\right)$ и $\mathcal{P}_{s-1}^{-}$в полупространстве $\Omega_{s-1}^{-}:\left(z-H_{s-1}<0\right)$, например

$$
\mathcal{P}_{s}^{+}(\mathbf{x}, \xi)=\frac{1}{2 \pi} \frac{\cos \left(\mathbf{x}-\xi, e_{z}\right)}{|\mathbf{x}-\xi|^{2}}
$$

$\mathbf{x} \in \Omega_{s}^{+}, \xi \in L_{s-1}$. Для любого измеримого множества $\omega \in L_{s-1}$ можно ввести индикатор $\chi_{\omega}(\xi)$ и гармоническую функцию

$$
\int_{L_{s-1}} \mathcal{P}_{s}^{+}(\mathbf{x}, \xi) \chi_{\omega}(\xi) d^{2} \xi=\int_{\omega} \mathcal{P}_{s}^{+}(\mathbf{x}, \xi) d^{2} \xi:=\mu^{\mathbf{x}}(\omega),
$$

называемую обычно гармонической мерой $\omega$ относительно точки х. Гармоническая мера пропорциональна пространственному углу, противолежашему множеству $\omega$, с коэффициентом $1 /(2 \pi)$. Этот геометрический факт будет использован в следуюшем разделе при получении оценок для отклонений приближенных решений поставленной выше задачи от приближенных решений, которые мы собираемся построить.

Можно ввести аналоги $\mu_{ \pm, s}^{\mathbf{x}}(\omega)$ гармонической меры на слоях. Например, если $\omega \in$ $L_{s-1}, x \in \Omega_{s}$, то

$$
\int_{\omega} \mathbf{P}_{s}^{+}(\mathbf{x}, \xi) d^{2} \xi:=\mu_{+, s}^{\mathbf{x}}(\omega) .
$$

Из принципа максимума следует, что аналоги отображения Пуассона на слоях доминируются отображениями Пуассона в полупространстве, например, если $\mathbf{x} \in \Omega_{s}, \omega \in$ $L_{s-1}$, имеем

$$
0<\mu_{+, s}^{\mathbf{x}}(\omega)<\mu_{+}^{\mathbf{x}}(\omega) .
$$

Для каждого диска $\omega_{s, t}$ рассмотрим цилиндр

$$
B_{s, t}=\left\{\mathbf{x} \in \omega_{s, t},\left|z-H_{s}\right|<\frac{1}{2} \min \left(d_{s}, d_{s+1}\right)\right\}
$$


разбиваемый диском $\omega_{s, t}$ на две равные части $B_{s, t}^{ \pm}$, которые лежат в верхнем и нижнем смежных слоях $\Omega_{s+1}, \Omega_{s}$, и соответствуюшие части гранишы $\Sigma_{s, t}^{ \pm} \in \partial B_{s, t}$. Сумма по всем верхним (нижним) частям цилиндров, основаниями которых являются диски $\omega_{s, t} \in L_{s}$, обозначим $B_{\omega_{s}}^{+}\left(B_{\omega_{s}}^{-}\right)$. Соответствующие множества на периоде обозначим $\mathbf{B}_{\omega_{s}}^{ \pm}$. Сходным образом можно построить цилиндры $B_{\gamma_{s}}^{+}, B_{\gamma_{s}}^{-}$с основаниями на $\gamma_{s}$. Обозначим через $\Sigma_{s, t}$ поверхность цилиндра $B_{s, t}$ и через $\Sigma_{s, t}^{ \pm}$верхнюю и нижнюю его части: $\Sigma_{s, t}^{ \pm} \subset \Omega_{s}^{ \pm}$. Можно определить также сумму поверхностей $\Sigma_{\omega_{s}}^{ \pm}=\bigcup_{t} \Sigma_{s, t}^{ \pm}$в целых бесконечных слоях или в их частях по периодам.

Получим теперь интегральное уравнение для решения первой из двух поставленных выше граничных задач (“антиточечная" проблема) на периоде с периодическими граничными условиями на вертикальной границе, условиями Дирихле на горизонтальных плоскостях $L_{s}$

$$
\Delta v=0,\left.\quad v\right|_{L_{s} \cap \Omega_{0}}=u_{s}, \quad s=0,1,2, \ldots, n,
$$

граничными условиями согласования на дисках $\omega_{s, t}$ и с условием Майкснера на границе дисков: $u \in W_{2}^{1}\left(\boldsymbol{\Omega}_{0}\right)$. Формально этот же метод может быть использован для решения второй ("квантово-точечной") проблемы, но решение промежуточной граничной задачи на $B_{\gamma_{s}}$ не может быть получено в точной форме.

Рассмотрим "антиточечную" задачу как возмущение исходной задачи с "бездырочностью" на пластинах $L_{s}$. Различие $w=v-u$ между решениями возмушенной и невозмушенной задач равно нулю на плоскостях вне дисков $\omega_{s, t}$. Мы можем восстановить гармоническую функцию $w$ в каждом слое $\Omega_{s}$, если известны ее сужения $w_{s, t}, w_{s-1, t}$ на диски $\omega_{s, t}, \omega_{s-1, t}$, расположенные внутри периода $\boldsymbol{\Omega}_{0}$ на плоскостях $L_{s-1}, L_{s}$. Выведем теперь интегральное уравнение для функций $w_{s, t}$. Обозначим через $\mathbf{w}$ столбец из вектор-функций $w_{s}, s=1,2, \ldots, n$, с компонентами $\left\{w_{s, t}\right\}, t=1,2, \ldots$ Для каждой вектор-функции $w_{s}$ можно построить периодические решения задачи Дирихле в слоях $\Omega_{s}, \Omega_{s+1}$, используя формулы для отображения Пуассона

$$
\begin{aligned}
& w_{s}^{+}(z)=\mathbf{P}_{s}^{+}\left[\sum_{t} w_{s, t}\right], \\
& w_{s}^{-}(z)=\mathbf{P}_{s-1}^{-}\left[\sum_{t} w_{s, t}\right] .
\end{aligned}
$$

Отсюда решение $w$ полной граничной задачи в слое $\Omega$ представляется в виде прямой суммы решений $w_{s}$ граничных задач в слоях $\Omega_{s}$ :

$$
\left.w\right|_{\Omega_{s}}=w_{s}=\mathcal{P}_{s}\left[\sum_{t} w_{s, t}+\sum_{t} w_{s-1, t}\right],
$$

и возмушенное решение в слое $\Omega_{s}$ равняется сумме невозмушенного кусочно-линейного решения (5) и корректируюшего дополнения $w: v=u+w$. Рассмотрим сужения построенного решения $u+w$ на поверхности $\Sigma_{\omega_{s, t}}$ цилиндров с основаниями на дисках $\omega_{s, t} \in L_{s}, \omega_{s-1, t} \in L_{s-1}$ :

$$
u+\left.w\right|_{\Sigma_{s, t}^{+}}: v_{\Sigma_{s, t}^{+}}, \quad u+\left.w\right|_{\Sigma_{s, t}^{-}}: v_{\Sigma_{s, t}^{-}}
$$


Тогда сужение решения $v$ возмушенной задачи (с дырами) на поверхность $\Sigma_{s, t}=\Sigma_{s, t}^{+} \cup$ $\Sigma_{s, t}^{-}$представляет собой прямую сумму сужений

$$
\left.v\right|_{\Sigma_{s, t}}=v_{\Sigma_{s, t}^{-}} \oplus v_{\Sigma_{s, t}^{+}} .
$$

Используя тот факт, что решение $v$ есть гармоническая функция внутри цилиндра $B_{s, t}$, ее можно представить внутри цилиндра $B_{s, t}$ посредством интеграла Пуассона:

$$
v=\mathcal{P}_{s, t}\left[v_{\Sigma_{s}^{-}} \oplus v_{\Sigma_{s}^{+}}\right]=\mathcal{P}_{s, t}\left[\left(u+\left.w\right|_{\Sigma_{s, t}^{+}}\right) \oplus\left(u+\left.w\right|_{\Sigma_{s, t}^{-}}\right)\right] .
$$

Теперь сужение полученной функции $v$ на диск $\omega_{s, t} \in B_{s, t}$ вновь дает начальную функцию $u_{s}+w_{s, t}$. Это приводит к интегральному уравнению для вектор-функции $w$. Описанный алгоритм построения уравнения для отклонения $w$ решения возмушенной граничной задачи от решения невозмушенной (без дыр) может быть представлен в операторной форме. Обозначим через $[\cdot]_{\omega}$ сужение выражения в скобках $[\cdot]$ на множество $\omega$. Тогда можно представить описанный алгоритм в следующей форме:

$$
\left[\mathcal{P}_{s, t}\left(\left[u+\widetilde{\mathbf{P}}_{s+1}\left(w_{s}+w_{s+1}\right)\right]_{\Sigma_{s, t}^{+}} \oplus\left[u+\widetilde{\mathbf{P}}_{s}\left(w_{s}+w_{s-1}\right)\right]_{\Sigma_{s, t}^{-}}\right)\right]_{\omega_{s, t}}=w_{s, t}+u_{s} .
$$

При некоторых условиях на геометрические параметры это уравнение может быть решено методом последовательных приближений (см. раздел 4). Чтобы описать процедуру построения приближенного решения, необходимо ввести нормы в пространстве непрерывных вектор-функций с компонентами, определенными на дисках и на слоях внутри периода $\boldsymbol{\Omega}_{0}$ :

$$
\left|w_{s}\right|=\max _{\mathbf{x}, t}\left|w_{s, t}(\mathbf{x})\right|, \quad|w|=\max _{s}\left|w_{s}\right| .
$$

Теорема 2. Уравнение (10) может быть представлено в операторной форме в пространстве непрерьвных функций на дисках

$$
w=K w+f,
$$

$2 \partial e$

$$
f=\left[\mathcal{P}_{s, t}\left([u]_{\Sigma_{s, t}^{-}} \oplus[u]_{\Sigma_{s, t}^{+}}\right)\right]_{\omega_{s, t}}=w_{s, t}
$$

u onepamop $K$,

$$
(K w)_{\omega_{s, t}}=\left[\mathcal{P}_{s, t}\left(\left[\widetilde{\mathbf{P}}_{s}\left(w_{s}+w_{s-1}\right)\right]_{\Sigma_{s, t}^{-}} \oplus\left[\widetilde{\mathbf{P}}_{s+1}\left(w_{s}+w_{s+1}\right)\right]_{\Sigma_{s, t}^{+}}\right)\right]_{\omega_{s, t}}=w_{s, t},
$$

является суммой сжимающего и компактного операторов в пространстве непрерывных функиий на дисках. В случае, когда в слой $\Omega$ введена лишь единственная пластина, оператор $K$ является компактным и решение уравнения (11) может бъть получено в форме сходящегося ряда

$$
w=f+K f+K^{2} f+K^{3} f+\cdots .
$$


ДокАЗАТЕльСтво. Отметим прежде всего, что оператор $K$ может быть представлен как сумма $K=K_{\text {diag }}+K_{\text {mix }}$ "диагонального" оператора

$$
\left(K_{\operatorname{diag}} w_{s}\right)_{\omega_{s, t}}=\left[\mathcal{P}_{s, t}\left(\left[\mathbf{P}_{s} w_{s}\right]_{\Sigma_{s, t}^{-}} \oplus\left[\mathbf{P}_{s+1} w_{s}\right]_{\Sigma_{s, t}^{+}}\right)\right]_{\omega_{s, t}},
$$

преобразуюшего функцию $w_{s}$, определенную на множестве дисков $\omega_{s}$, в функцию, определенную на том же множестве дисков, и "перемешиваюшего" оператора

$$
\left(K_{\text {mix }}\left(w_{s-1}+w_{s+1}\right)\right)_{\omega_{s, t}}=\left[\mathcal{P}_{s, t}\left(\left[\mathbf{P}_{s} w_{s-1}\right]_{\Sigma_{s, t}^{-}} \oplus\left[\mathbf{P}_{s+1} w_{s+1}\right]_{\Sigma_{s, t}^{+}}\right)\right]_{\omega_{s, t}}=w_{s, t}
$$

преобразуюшего функцию $w_{s \pm 1}$, определенную на множестве дисков $\omega_{s-1}, \omega_{s+1}$, в функцию, определенную на множестве дисков $\omega_{s}$. Видно, что перемешиваюший оператор содержит сужения отображений Пуассона $\mathbf{P}_{s}^{-}, \mathbf{P}_{s-1}^{+}$, действуюших на функции $w_{s+1}$, $w_{s-1}$, соответственно, на поверхности $\Sigma_{s, r}$ вершин цилиндров $\min \left(d_{s} / 2, d_{s+1} / 2\right):=\delta_{s}$ с основаниями на пластине $L_{s}$. Из формулы (7) можно получить экспоненциальную оценку для сужений, например

$$
\begin{aligned}
\left.\mathbf{P}_{s}^{-} w_{s+1}\right|_{\Sigma_{s, t}^{+}} & =\left.\sum_{l} \varphi_{l}(\mathbf{x}) u_{s+1, l} \frac{\operatorname{sh}|l|\left(z-d_{s}\right)}{\operatorname{sh}|l| d_{s+1}}\right|_{\mathbf{x}, z \in \Sigma_{s, t}^{+}}, \\
\left|\mathbf{P}_{s}^{-} w_{s+1}\right|_{\Sigma_{s, t}^{+}} \mid & \leqslant \sum_{l} \frac{l}{\sqrt{D_{1} D_{2}}}\left|u_{s+1, l}\right| \frac{\operatorname{sh}|l|\left(d_{s+1}-\delta_{s}\right)}{\operatorname{sh}|l| d_{s+1}},
\end{aligned}
$$

или даже

$$
\left.\mathbf{P}_{s}^{-} w_{s+1}\right|_{\Sigma_{s, t}^{+}}=\left.\sum_{l \leqslant M} \varphi_{l}(\mathbf{x}) u_{s+1, l} \frac{\operatorname{sh}|l|\left(z-H_{s}\right)}{\operatorname{sh}|l| d_{s+1}}\right|_{\mathbf{x}, z \in \Sigma_{s, t}^{+}}+O\left(e^{-M\left[\delta_{s}\right]}\right),
$$

где через $O\left(e^{-M\left[\delta_{s}\right]}\right)$ обозначен малый оператор с нормой, оцениваемой соответствующей экспонентой с некоторым положительным множителем. Принимая во внимание, что первое дополнение в правой части формулы (14) есть конечномерный оператор, заключаем, что вышеуказанное сужение, равно как и весь оператор $K_{\text {mix }}$, является компактным оператором в пространстве непрерывных функций:

$$
K_{\mathrm{mix}}=K_{\mathrm{mix}}^{M}+K_{\mathrm{mix}}^{\varepsilon}
$$

где $M$-мерная часть обозначена $K_{\text {mix }}^{M}$ и малая часть обозначена $K_{\mathrm{mix}}^{\varepsilon}$. Отметим, что выполненные вычисления дают также связь между $M$ и $\varepsilon$.

Исследуем теперь диагональную часть оператора $K$. Обозначим $\chi_{s-1, t}$ индикатор диска $\omega_{s-1, t}$ :

$$
\chi_{s-1, t}(\mathbf{x})= \begin{cases}1, & \mathbf{x} \in \omega_{s-1, t} \\ 0, & \mathbf{x} \in L_{s} \backslash \omega_{s-1, t}\end{cases}
$$

и рассмотрим граничную задачу для уравнения Лапласа в слое $\Omega_{s}$ с граничным условием $w_{s-1, t}=\chi_{s-1, t}$ на всем семействе дисков на пластине $L_{s-1}$. Благодаря принципу максимума решение $\chi_{s}$ такой граничной задачи строго менше, чем решение внутри 
слоя $\Omega_{s}$. Более того, сужение решения на каждую поверхность $\Sigma_{s-1, t}$ строго меньше, чем

$$
\left.\max _{s, t} \chi\right|_{\Sigma_{s-1, t}}:=\mu_{s-1}
$$

Чтобы удостовериться в этом, необходимо вычислить предел сужения при приближении к пластине $L_{s-1}$, поскольку значения решений внутри слоя $\Omega_{s}$ между пластинами $L_{s}, L_{s-1}$ строго меньше единицы. Мы оцениваем предел решения задачи Дирихле в слое $\Omega_{s}$ с граничными данными на верхней границе пластины $L_{s-1}$ решением задачи Дирихле в полупространстве $\Omega_{s}^{+}$, которое равно гармонической мере $\mu^{\mathbf{x}}\left(\omega_{s}\right)$ всей периодической системы дисков. При приближении пластины $L_{s-1} \mathrm{k}$ границе диска $\omega_{s, t}$ видно, что гармонические меры всех других дисков $\mu^{\mathbf{x}}\left(\omega_{s, t^{\prime}}\right), t^{\prime} \neq t$, стремятся к нулю благодаря равномерной сходимости ряда

$$
\frac{1}{2 \pi} \sum_{\mathbf{m} \neq 0} \frac{\pi \delta^{2}\left(z-H_{s-1}\right)}{\left[\left(m_{1} D_{1}\right)^{2}+\left(m_{2} D_{2}\right)^{2}+\left(z-H_{s-1}\right)^{2}\right]^{3 / 2}},
$$

асимптотически приближаясь к гармонической мере объединения всех нетривиальных периодических сдвигов одного диска $\omega_{s, r}$. Поскольку полное число $M_{s-1}$ дисков на

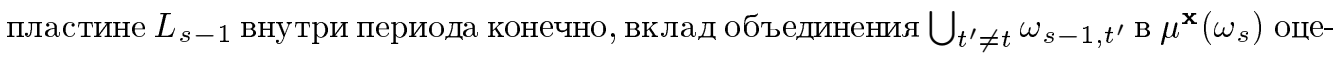
нивается как

$$
\frac{M_{s-1}}{2 \pi} \sum_{\mathbf{m} \neq 0} \frac{\pi \delta^{2}\left(z-H_{s-1}\right)}{\left[\left(m_{1} D_{1}\right)^{2}+\left(m_{2} D_{2}\right)^{2}+\left(z-H_{s-1}\right)^{2}\right]^{3 / 2}}
$$

и стремится к нулю, когда $z \rightarrow H_{s-1}$.

Оставшийся член в $\mu^{\mathbf{x}}\left(\omega_{s}\right)$, обусловленный наличием диска $\omega_{s, t}$, когда точка $(\mathbf{x}, z)$ приближается к $\left(\mathbf{x}, H_{s-1}\right)$, может быть оценен интегралом

$$
\mu_{0}=\frac{1}{2 \pi} \int_{\omega_{s, t}} \frac{z-H_{s-1}}{|| \boldsymbol{\xi}-\left.\mathbf{x}\right|^{2}+\left.\left|z-H_{s-1}\right|^{2}\right|^{3 / 2}} d \boldsymbol{\xi}^{2}
$$

который мажорируется схожим интегралом по полудиску радиуса $2 \delta$ с центром в точке $\mathbf{x}$ и может быть легко сосчитан:

$$
\mu_{0}<\frac{1}{2}\left[1-\frac{z-H_{s-1}}{\sqrt{\left|z-H_{s-1}\right|^{2}+\delta^{2}}}\right] \rightarrow \frac{1}{2}
$$

при $z-H_{s-1} \rightarrow 0$. Следовательно, сужение гармонической меры суммы дисков $\omega_{s-1}$ на сумму поверхностей $\Sigma_{s-1, t}^{+}$строго меньше единицы:

$$
\left.\sup \mu_{s}\right|_{\Sigma_{s-1, t}^{+}}:=\mu_{s-1}^{+}<1
$$

Объявленная оценка для нормы диагональной части оператора $K$ может быть получена из этого факта на основе принципа максимума. Чтобы удостовериться в этом, положим, что норма граничных данных $w$ в пространстве непрерьвных функций есть $\|w\|$. Тогда $\left.|w|\right|_{\omega_{s-1, t}}<\|w\| \chi_{s-1, t}$, и “диагональная" часть решения $\mathbf{w}$ подходящей задачи 
Дирихле может быть оценена на поверхностях $\Sigma_{s-1, t}^{+}$благодаря принципу максимума как

$$
\sup _{\Sigma_{s-1, t}^{+}}|\mathbf{w}| \leqslant \mu_{s-1}\|w\| .
$$

Если сходная оценка имеет место в соседнем слое $\Omega_{s-1}$, тогда мы можем оценить решение граничной задачи с данными $|w|$ на всей поверхности $\Sigma_{s-1, t}=\Sigma_{s-1, t}^{+} \cup \Sigma_{s-1, t}^{-}$через $\max \left[\mu_{s-1}^{+}, \mu_{s-1}^{-}\right]\|w\|$. Затем согласно принципу максимума, применив отображение Пуассона к граничньмм данным на всей поверхности $\Sigma_{s, t}$, получим оценку для решения граничной задачи внутри цилиндра $B_{s-1, t}$ :

$$
|\mathbf{w}| \leqslant \max \left[\mu_{s-1}^{+}, \mu_{s-1}^{-}\right]\|\mathbf{w}\| .
$$

Следовательно, сужение этого решения на диск $\omega_{s-1, t} \in B_{s-1, t}$ также удовлетворяет оценке вьше. Теперь диагональная часть оператора $K$ является сжимающей, поскольку $\max \left[\boldsymbol{\mu}_{s-1}^{+}, \boldsymbol{\mu}_{s-1}^{-}\right]<1$ для любого $s=2,3, \ldots, n$. Теорема доказана.

ЗАмЕчАНИЕ. Отметим, что оценки для гармонической меры в теореме 2 могут быть слегка модифицированы, чтобы включить оценку вклада дисков с другой пластины. Чтобы удостовериться в этом, обозначим через $\mu_{s-1}$ максимальное значение гармонической меры суммы дисков $\omega_{s}$ на поверхностях $\Sigma_{s-1, t}^{+}$с основаниями на дисках $\omega_{s-1, t}$, расположенных на пластине $L_{s-1}$. Тогда из оценок, полученных выше, следует, что $\mu_{s-1}$ мажорируется рядом

$$
\frac{M_{s}}{2 \pi} \sum_{\mathbf{m}} \frac{\pi \delta^{2} d_{s}}{\left[\left(m_{1} D_{1}\right)^{2}+\left(m_{2} D_{2}\right)^{2}+\left(d_{s}-\delta_{s}\right)^{2}\right]^{3 / 2}}:=\mu_{s} .
$$

Если сумма $\mu_{s-1}+\mu_{s}$ строго меньше единицы, то сужение на $\Sigma_{s-1, t}^{+}$гармонической меры всего образца дисков $\omega_{s} \subset L_{s}$ и $\omega_{s-1} \subset L_{s-1}$ строго меньше единицы. В следуюшем разделе приведены некоторые грубые оценки для максимумов гармонической меры, которые могут гарантировать сходимость ряда последовательных приближений (12).

\section{4. АПРИОРНЫЕ ОЦЕНКИ}

Используем итерационные решения, полученные вьше, как аппроксимации для точного решения задачи. Хотя наша итерационная процедура является сходяшейся геометрически, необходимы предварительные оценки отклонений конечных аппроксимаций от точного решения. Эти оценки могут быть получены из следуюших нескольких элементарных утверж дений.

Лемма 1. Если области $\omega_{s}, \quad s=1,2$, компактны, $B_{s}$ - минимальные шары, содержащие $\omega_{s}, \operatorname{diam} B_{s}=2 d_{s} u \operatorname{dist}\left(\omega_{1}, \omega_{2}\right)=d, m o$

$$
0<\sup _{\Gamma_{2}} \xi_{1}<\frac{d_{1}}{d+d_{1}}, \quad 0<\sup _{\Gamma_{1}} \xi_{2}<\frac{d_{2}}{d+d_{2}},
$$

и норма оператора $K$ из раздела 3 может быть оценена как

$$
\|K\| \leqslant \frac{d_{1} d_{2}}{\left(d+d_{2}\right)\left(d+d_{1}\right)} .
$$


ДокАЗАТЕЛЬСТво основывается на принципе максимума: функции $\xi_{s}$ мажорируются в $\Omega_{s}$ кулоновскими потенциалами зарядов $4 \pi d_{s}$, расположенных в центрах минимальных шаров $B_{s}$, содержащих $\omega_{s}$.

ЛЕмма 2. Предположим, что область $\omega$ компактна, В - минимальный шар, содержащий $\omega, \operatorname{diam} B=2 d u \Gamma_{1,2}$ - параллельные плоскости, образующие открытый слой $\Omega$, ограниченный $\Gamma_{1,2}, \operatorname{dist}\left(\Gamma_{1,2}, B_{1}\right)=d_{1,2}$. Рассмотрим отображсение Пуассона $\mathcal{P}$ дополнительной области $\mathbb{R}^{3} \backslash \omega$ и отображсение Пуассона $\mathcal{P}_{\Gamma}$ невозмущенного слоя $\Omega$. Рассмотрим задачу Дирихле в возмущенном слое $\Omega \backslash \omega$ снулевыми граничными условиями на плоскостях и константой $\left.\right|_{\Gamma}=u_{\Gamma}$ на границе $\partial \omega=\Gamma$. Тогда норма интегрального оператора в $C_{\Gamma}$

$$
K: u \rightarrow\left[\mathcal{P}_{\Gamma}[\mathcal{P} u]_{\Gamma_{1} \cup \Gamma_{2}}\right]_{\Gamma}
$$

оценивается как

$$
\|K\| \leqslant \sup _{x \in \Gamma} \leqslant \frac{d}{d_{1}+d} \frac{\operatorname{dist}\left(x, \Gamma_{2}\right)}{d_{1}+d_{2}+2 d}+\frac{d}{d_{2}+d} \frac{\operatorname{dist}\left(x, \Gamma_{1}\right)}{d_{1}+d_{2}+2 d} .
$$

ДоКАЗАТЕЛЬСТВо основывается на принципе максимума: соответствующие функции $\xi_{s}$ мажорируются в $\Omega$ кулоновским потенциалом заряда $4 \pi d$, расположенного в центре минимального шара $B$, содержащего $\omega$, и линейной функцией внутри невозмушенного слоя.

ЛЕмма 3. Сужения гармонических функиий $\mathcal{P}_{i} \chi_{s, t}, i=s-1, s$, на каждую полусферу $\Sigma_{i, t}^{ \pm}$допускает оценку

$$
\sup _{\Sigma_{i, t}^{ \pm}}\left|\mathcal{P}_{i} \chi_{s, t}\right| \leqslant 1-\frac{1}{\sqrt{2}}
$$

ДокАЗАТЕЛЬСТво можно провести с использованием того факта, что решение граничной задачи внутри слоя в силу принципа максимума не превышает решения граничной задачи с теми же граничными данными $\chi_{s, t}$ в соответствуюшем полупространстве. Утверждение остается справедливым при замене одиночной граничной функции $\chi_{s, t}$ на плоскости $L_{s}$ соответствуюшей периодически продолженной функцией $\tilde{\chi}_{s, t}$. Решения $v_{s}^{+}, v_{s}^{-}$граничной задачи в смежных полупространствах $\Omega_{s}^{+}, \Omega_{s}^{-}$, соответственно, и $\tilde{v}_{s+1}^{+}, \tilde{v}_{s}^{-}$в слоях $\Omega_{s+1}, \Omega_{s}$ с периодически продолженными граничными условиями являются положительными периодическими функциями, $0<\tilde{v}_{s+1}<v_{s+1}, 0<\tilde{v}_{s}<v_{s}$, поскольку $\tilde{v}_{s-1}, \tilde{v}_{s}$ исчезают на $L_{s-1}, L_{s+1}$, соответственно. Мы оцениваем решение $v_{s}$ в полупространстве $\Omega_{s}^{+}$, основываясь на факте, что, вообще говоря, решение задачи Дирихле с граничными данными, положенными равными индикатору $\chi_{\omega}$ множества $\omega \in L_{s}$ (гармоническая мера множества, сосчитанная в точке $\mathbf{x}$ ), равняется пространственному углу в точке $\mathbf{x}$, противолежашему множеству $\omega$ с коэффициентом $(4 \pi)^{-1}$. В 
нашем случае множество представлено как сумма $\omega_{s, t}$ и сдвигов на нем. Пространственный угол в северном полушарии $B_{s, t}$, под которым видно множество $\omega_{s, t}$, равняется $4 \pi(1 / 2-1 /(2 \sqrt{2}))$, а угол, под которым видны все периодические сдвиги $\omega_{s, t}$, асимптотически мажорируется сходящимся рядом и оценивается следующим образом:

$$
2 \pi \delta^{3} \sum_{\mathbf{m} \neq 0} \frac{1}{\left[\left(m_{1} D_{1}\right)^{2}+\left(m_{2} D_{2}\right)^{2}+\delta^{2}\right]^{3 / 2}} \leqslant 2 \pi .
$$

Суммируя вышеизложенные результаты, можно оценить гармоническую меру множества всех сдвигов $m \in \mathbb{Z}_{2}$ дисков $\omega_{s, t}$ на северном полюсе шара $B_{s, t}$ как

$$
\frac{1}{4 \pi}[2 \pi+2 \pi-\pi \sqrt{2}]=1-\frac{1}{2 \sqrt{2}}<1 .
$$

Оценка ограничения на полусферу $\Sigma_{+}$решения $\tilde{v}_{s}$ в слое $\Omega_{s}$ следует из полученного выше неравенства для гармонической меры $\tilde{v}$ в силу принципа максимума. Лемма доказана.

СЛЕДСТВИЕ. Предполагая, что имеется несколько дисков $\omega_{s, t} \in L_{s}$ на периоде $\boldsymbol{\Omega}_{0}$, можсно оченить угол в точке на сумме полусфер $\sum_{t} \Sigma_{s, t}$ с основаниями

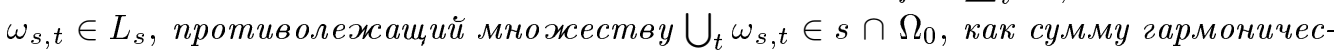
кой меры одного диска и гармонической мерь всех других дисков, поэтому гармоническая мера сумми $\sum_{t} \omega_{s, t}$ на полусферах $\sum_{t} \Sigma_{s, t}$ допускает ту же оценку, а именно $1-(2 \sqrt{2})^{-1}$. Следовательно, результат не зависит от радиусов $\delta_{s, t}$ дисков $\omega_{s, t}$ и их числа, если диски не перекрываются $и \sup _{t} \delta_{s, t}<\min \left[\left(d_{s+1}-d_{s}\right)\right.$, $\left.\left(d_{s}-d_{s-1}\right)\right]$.

Чтобы оценить гармоническую меру дисков $\sum_{t} \omega_{s, t} \in L_{s}$ на полусферах $\sum_{t} \Sigma_{s+1, t}$ с основаниями на дисках $\sum_{t} \omega_{s+1, t} \in L_{s+1}$, необходимы дополнительные данные о геометрической конфигурации плоскостей $L_{s}, L_{s+1}$ и дисков на них. Следующее утверждение почти очевидно.

ЛЕмма 4. Рассмотрим граничную задачу для уравнения Лапласа на доле $\Omega_{s}$ периода $\Omega_{0}$ с периодическими граничными условиями на вертикальной граниче и граничными данными $\sum_{t} \chi_{s, t}$ на верхней крышке $L_{s} \cap \boldsymbol{\Omega}_{0}$. Тогда максимум решения $\tilde{v}_{s}$ на сумме полусфер $\sum_{t} \Sigma_{s-1, t}$ с основаниями на $\sum_{t} \omega_{s-1, t}$, может бъть оченен как

$$
\max \tilde{v}_{s} \leqslant \frac{\sum_{t} \delta_{s, t}^{2}}{2\left(d_{s}-d_{s-1}-\max _{t} \delta_{s-1, t}\right)^{2}}:=\rho_{s-1,+} .
$$

Аналогичная оценка имеет место для максимума гармонической мерь дисков $\sum_{t} \omega_{s-1, t}$ на полусферах, находящихся на дисках $\sum_{t} \omega_{s, t}$ :

$$
\max \tilde{v}_{s-1} \leqslant \frac{\sum_{t} \delta_{s-1, t}^{2}}{2\left(d_{s}-d_{s-1}-\max _{t} \delta_{s, t}\right)^{2}}:=\rho_{s,-} .
$$


Суммируя полученные результаты, можно сформулировать следующую априорную оценку для решения $v$ граничной задачи в $\Omega_{s} \cap \boldsymbol{\Omega}_{0}$ с периодическими граничными данными на вертикальной границе и условиями Дирихле на верхней и нижней крышках $L_{s} \cap \boldsymbol{\Omega}_{0}, L_{s-1} \cap \boldsymbol{\Omega}_{0}:$

$$
\left.v\right|_{L_{s} \cap \boldsymbol{\Omega}_{0}}=\sum_{t} \chi_{s, t},\left.\quad v\right|_{L_{s-1} \cap \boldsymbol{\Omega}_{0}}=\sum_{t} \chi_{s-1, t}
$$

Теорема 3. Решение указанной граничной задачи допускает оценки

$$
\begin{gathered}
\left.v\right|_{\sum_{t} \Sigma_{s, t}} \leqslant 1-\frac{1}{2 \sqrt{2}}+\rho_{s,-}, \\
\left.v\right|_{\sum_{t} \Sigma_{s-1, t}} \leqslant 1-\frac{1}{2 \sqrt{2}}+\rho_{s-1,+}
\end{gathered}
$$

на семействе полусфер $\sum_{t} \Sigma_{s, t}, \sum_{t} \Sigma_{s-1, t}$ с основаниями на дисках $\omega_{s, t} \in L_{s} u$ $\omega_{s-1, t} \in L_{s-1}$, соответственно.

Благодарности. Авторы признательны профессору В. Бабичу за важные ссылки и профессору С. М. Ермакову за интересные и вдохновляющие дискуссии.

\section{Список литературы}

[1] V. Ryzhii. Semicond. Sci. Technol. 1996. V. 11. P. 759-765.

[2] V. Ryzhii, V. Pipa, I. Khmyrova, V. Mitin, M. Wilander. Japan. J. Appl. Phys. P. 2. 2000. V. 39. P. L1283-L1285.

[3] M. Ryzhii, V. Ryzhii, V. Mitin. Microelectronics J. 2003. V. 34. P. 411-414.

[4] И. И. Ворович, В. М. Александров, В. А. Бабешко. Неклассические смешанные задачи теории упругости. М.: Наука, 1974

[5] В. Готлиб. ДАН СССР. 1986. Т. 287. № 6. С. 1109-1113.

[6] B. Pavlov. New Zealand J. Math. 1996. V. 25. P. 199-216.

[7] H. Н. Лебедев. Специальные функции в математической физике. М.: Наука, 1986.

Поступила в редакцию 22.IX.2003 г., после доработки 18.II.2004 г. 